\title{
CONCAVITY OF POWERS OF A CONVOLUTION
}

\author{
DOUGLAS HENSLEY
}

\begin{abstract}
A short geometric proof of a result of Brascamp and Lieb on concavity of powers of a convolution is given.
\end{abstract}

The following theorem, a special case of Corollary 3.5 of [2], has a very simple proof for positive integers $p$ and $q$.

Let $f, g: \mathbf{R}^{n} \rightarrow \mathbf{R}^{+} \cup\{0\}$ be positive on bounded convex sets $S(f), S(g)$ respectively and 0 elsewhere. Let $p$ and $q$ be positive integers.

THEOREM 1. If $f^{1 / p}$ and $g^{1 / q}$ are concave on their respective, convex supports then $(f * g)^{1 /(p+q+n)}$ is concave on the Minkowski set sum $S(f)+S(g)$ and 0 elsewhere.

REMARK. Suitably interpreted this also holds if $p$ or $q$, or both, are zero. The exponent $1 /(p+q+n)$ is best possible, in all cases.

Proof OF THEOREM 1. Let $F \subseteq \mathbf{R}^{p+n}=\left\{\left(x_{1}, x_{2} \cdots x_{n}, w_{1}, w_{2} \cdots w_{p}\right):\left|w_{i}\right|\right.$ $<\frac{1}{2} f^{1 / p}\left(x_{1}, x_{2} \cdots x_{n}\right)$ for $\left.1<i<p\right\}$. Let

$$
\begin{aligned}
G & \subseteq \mathbf{R}^{q+n} \\
& =\left\{\left(y_{1}, y_{2} \cdots y_{n}, v_{1}, v_{2} \cdots v_{q}\right):\left|v_{i}\right|<\frac{1}{2} g^{1 / q}\left(y_{1} \cdots y_{n}\right) \text { for } 1<i<q\right\} .
\end{aligned}
$$

Then $F, G$ and $F \times G$ are convex sets.

Denote $\left(x_{1} \cdots x_{n}, w_{1} \cdots w_{p}, y_{1} \cdots y_{n}, v_{1} \cdots v_{q}\right)$ by $(\bar{x}, \bar{w}, \bar{y}, \bar{v})$ and for $\bar{z} \in$ $\mathbf{R}^{n}$ let $H_{\bar{z}}$ be the affine space $\{(\bar{x}, \bar{w}, \bar{y}, \bar{v}): \bar{x}+\bar{y}=\bar{z}\}$. Let $J_{\bar{\alpha}}$ be the affine space $\{(\bar{x}, \bar{w}, \bar{y}, \bar{v}): \bar{x}=\bar{\alpha}\}$. For arbitrary $\bar{z}$ and $\bar{\alpha}, F \times G \cap H_{\bar{z}} \cap J_{\bar{\alpha}}$ is either empty or the Cartesian product of a $p$-dimensional open cube of volume $f(\bar{\alpha})$ with a $q$-dimensional open cube of volume $g(\bar{z}-\bar{\alpha})$. In either case the $(p+q)$ dimensional volume of $H_{\bar{z}} \cap J_{\bar{\alpha}} \cap F \times G$ is $f(\bar{\alpha}) g(\bar{z}-\bar{\alpha})$.

Since $H_{\bar{z}}$ is the disjoint union of the $H_{\bar{z}} \cap J_{\bar{\alpha}}$ and the distance between flats $H_{\bar{z}} \cap J_{\bar{\alpha}}$ and $H_{\bar{z}} \cap J_{\bar{\alpha}^{\prime}}$ is $\sqrt{2}\left\|\bar{\alpha}-\bar{\alpha}^{\prime}\right\|$, the $(p+q+n)$-dimensional volume $V(\bar{z})$ of $F \times G \cap H_{\bar{z}}$ is given by

$$
V(z)=(\sqrt{2})^{n} \int_{\bar{\alpha} \in \mathbf{R}^{n}} f(\bar{\alpha}) g(\bar{z}-\bar{\alpha}) d \bar{\alpha}=(\sqrt{2})^{n}(f * g)(\bar{z}) .
$$

Let us fix $z_{2}, \ldots, z_{n}$ and consider $V(\bar{z})$ as a function of $z_{1}$ alone.

Since the choice of coordinate axes is arbitrary it will suffice to prove that $V(\bar{z})^{1 /(p+q+n)}$ is concave in $z_{1}$ on the interval where it is positive.

Received by the editors April 1, 1980 and, in revised form, August 15, 1980.

AMS (MOS) subject classifications (1970). Primary 52A40, 52A20, 60D05. 
Let $H^{\prime}=\left\{(\bar{x}, \bar{w}, \bar{y}, \bar{v}): x_{i}+y_{i}=z_{i}\right.$ for $\left.2<i<n\right\}$, and let $H^{\prime \prime}\left(z_{1}\right)=$ $\left\{(\bar{x}, \bar{w}, \bar{y}, \bar{v}): x_{1}+y_{1}=z_{1}\right\}$. Then $F \times G \cap H^{\prime}$ is convex and $H^{\prime \prime}\left(z_{1}\right)$ is a hyperplane in $H^{\prime}$. Let $\phi\left(z_{1}\right)=V^{1 /(p+q+n)}$ where $V$ is the $(p+q+n)$-dimensional volume of $\left(F \times G \cap H^{\prime}\right) \cap H^{\prime \prime}\left(z_{1}\right)$. Then by the Brunn-Minkowski theorem, $\phi\left(z_{1}\right)$ is concave on its support.

\section{REFERENCES}

1. C. Borell, Convex set functions in d-space, Period. Math. Hungar. 6 (1975), 111-136.

2. H. J. Brascamp and E. H. Lieb, On extensions of the Brunn-Minkowski and Prékopa-Leindler theorems, J. Funct. Anal. 22 (1976), 366-389.

3. J. S. Davidovic, B. I. Korenbljum and B. I. Hacet, A property of logarithmically concave functions, Dokl. Akad. Nauk SSSR 185 (1969); Soviet Math. Dokl. 10 (1969), 477-480.

4. H. Eggleston, Convexity, Cambridge Univ. Press, London and New York, 1958, p. 97.

5. I. A. Ibragimov, On the composition of unimodal distributions, Teor. Verojatnost. i Primenen. 1 (1956), 283-288.

6. C. G. Lekkerkerker, A property of logarithmic concave functions, Indag. Math. 15 (1953), 505-521.

7. A. Prékopa, On logarithmic concave measures and functions, Acta Sci. Math. (Szeged) 34 (1973), 335-343.

8. Y. Rinoth, On convexity of measures, Ann. Probab. 4 (1976), 1020-1026.

Department of Mathematics, Texas adM University, College Station, Texas 77843 